
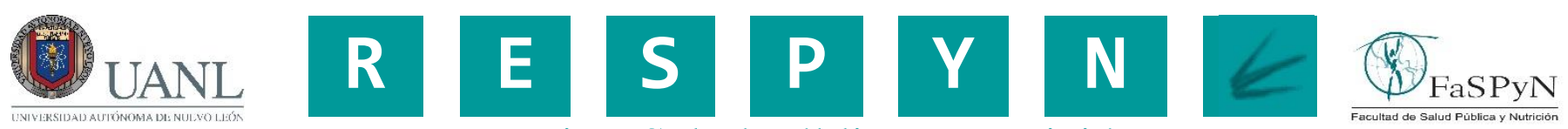

Revista Salud Pública y Nutrición

\title{
ENFOQUES Y FACTORES ASOCIADOS A LA INSEGURIDAD ALIMENTARIA.
}

\author{
APPROACHES AND FACTORS ASSOCIATED WITH FOOD INSECURITY.
}

Pérez Garcés Ranulfo ${ }^{1}$, Silva Quiroz Yolanda ${ }^{2}$.

1 Universidad Autónoma del Estado de México, México. 2 Consultor independiente, México.

Citation: Pérez Garcés R., Silva Quiroz Y. (2019) Enfoques y factores asociados a la inseguridad alimentaria. Revista de Salud Pública y Nutrición, 18 (1), 1524.

Editor: Esteban G. Ramos Peña, Dr. CS., Universidad Autónoma de Nuevo León, Facultad de Salud Pública y Nutrición, Monterrey Nuevo León, México. Copyright: (C2019 Pérez Garcés R. et al. This is an open-access article distributed under the terms of Creative Commons Attribution License [CC BY 4.0], which permits unrestricted use, distribution, and reproduction in any medium, provided the original author and source are credited.

Competing interests: The authors have declared that no competing interests exist.

DOI: https://doi.org/10.29105/respyn18.1-3

Recibido: 27 de noviembre 2018; Aceptado: 20 de marzo 2019

Email: ranulfoprez121@gmail.com 


\title{
ENFOQUES Y FACTORES ASOCIADOS A LA INSEGURIDAD ALIMENTARIA
}

\author{
Pérez Garcés Ranulfo ${ }^{1}$, Silva Quiroz Yolanda ${ }^{2}$.
}

1 Universidad Autónoma del Estado de México, México. 2 Consultor independiente, México.

\section{RESUMEN}

Introducción En este artículo se ofrece una revisión de los principales enfoques teóricos utilizados tanto en México como en otros países para el estudio de la seguridad alimentaria y nutricional en población infantil; destacando en cada uno de ellos, los factores asociados y resultados más relevantes. Objetivo: Realizar un balance sobre la literatura existente a fin de identificar los principales enfoques teóricos y los factores asociados a la seguridad e inseguridad alimentaria. Material y Métodos: La búsqueda de trabajos se realizó en tres bases de datos de revistas científicas electrónicas. La selección se realizó con base en los siguientes criterios: a) que el tema principal fuese la seguridad alimentaria, b) que estudiaran la seguridad alimentaria de población menor de 18 años, c) que estudiaran la seguridad alimentaria en hogares y d) que en los resultados se hiciera referencia a hallazgos específicos menores de 11 años. Los trabajos revisados se organizaron en torno a cuatro enfoques principales: el enfoque neoclásico, el enfoque biomédico, el enfoque sociológico y el enfoque de modos de vida sostenibles. Resultados: Se encontraron 24 artículos que abordan los cuatro enfoques Conclusiones: La revisión bibliográfica realizada permite plantear que la seguridad alimentaria al ser un fenómeno complejo y multidimensional, no puede ser abordado en su totalidad desde una única perspectiva teórica, sino que se requiere cada vez más de enfoques holísticos y multidisciplinarios para su abordaje.

Palabras Clave: Inseguridad alimentaria, enfoques teóricos, seguridad alimentaria y nutricional.

\section{ABSTRACT}

Introduction: This article offers a review of the main theoretical approaches used in Mexico and other countries for the study of child food and nutrition security; highlighting in each of them, the associated factors and most relevant results. Objective: To make a balance on the existing literature in order to identify the main theoretical approaches and the factors associated with food security and insecurity. Methods: The search for works was carried out in three databases of electronic scientific journals. The selection was made based on the following criteria: a) what the main topic was food security, b) to study the food security of the population under 18 years of age, c) to study food security in households and point out findings about children. The reviewed works were organized around four main approaches: the neoclassical approach, the biomedical approach, the sociological approach and the sustainable livelihoods approach. Results: We found 24 articles that address the four approaches. Conclusions: The bibliographic review carried out allows us to state that food security, being a complex and multidimensional phenomenon, this cannot be analyzed from a single theoretical lens, but increasingly requires holistic and multidisciplinary approaches.

Key words: Food insecurity, theoretical approaches, food and nutritional security 


\section{Introducción}

Erradicar el hambre se posicionó dentro de la Agenda internacional en 1974, en el marco de la Conferencia Mundial de la Alimentación realizada en Roma, Italia. Posteriormente, durante la Cumbre Mundial sobre la Alimentación de 1996 se planteó que el problema del hambre era mucho más complejo, y se optó por orientar las acciones de los Estados hacia el logro de la seguridad alimentaria; la cual existía "cuando todas las personas tienen en todo momento acceso físico y económico a suficientes alimentos inocuos y nutritivos para satisfacer sus necesidades alimenticias y sus preferencias en cuanto a los alimentos a fin de llevar una vida activa y sana" (FAO, 1996); cuyas estrategias prioritarias serían garantizar la disponibilidad de alimentos, la estabilidad social y política tanto a nivel nacional como internacional, aumentar la producción de alimentos y hacer uso de los excedentes (FAO, 1996).

En 2015 se fijó como uno de los Objetivos de Desarrollo Sostenible a la seguridad alimentaria y nutricional. El Objetivo 2 de la Agenda 2030 planteó "Poner fin al hambre, lograr la seguridad alimentaria y la mejora de la nutrición y promover la agricultura sostenible". Sin embargo, en el informe Panorama de la seguridad alimentaria y nutricional de América Latina y el Caribe 2017. Sistemas alimentarios sostenibles para poner fin al hambre y la malnutrición, se subrayó que el hambre en el mundo, incluida América Latina, estaba aumentando; y que en México aun cuando se producían alimentos suficientes para alimentar a la población, ello no garantizaba que el consumo fuese adecuado, variado y nutritivo (Organización de las Naciones Unidas para la Alimentación y la Agricultura [FAO] y Organización Panamericana de la Salud [OPS], 2017).

A partir de este contexto, en el marco de un proyecto más amplio sobre seguridad alimentaria de población infantil en el Estado de México, se planteó como un objetivo prioritario realizar un balance sobre la literatura existente sobre el tema, a fin de identificar los principales enfoques teóricos y los factores asociados a la seguridad e inseguridad alimentaria, así como para identificar las líneas de investigación aún pendientes de abordar.

\section{Material y Métodos}

El presente estudio es tipo descriptivo y analítico se realizó mediante la revisión de artículos científicos sobre la seguridad alimentaria en población infantil en México principalmente y otras regiones. Se incluyeron trabajos de corte cualitativo y cuantitativo, publicados e indexados en los últimos cinco años, o más, si su relevancia era mencionada por otros autores. La búsqueda de trabajos se realizó principalmente en las plataformas de Ebesco, Scielo y Redalyc, mediante el uso de las frases clave "food security, "food insecurity", "Childhood food insecurity" "Seguridad alimentaria", "Seguridad alimentaria y nutricional", "Seguridad alimentaria en niños" y "Seguridad alimentaria infantil".

La selección de artículos se realizó con base en los siguientes criterios: a) qué el tema principal fuese la seguridad alimentaria, b) que estudiaran la seguridad alimentaria de población menor de 18 años, c) que estudiaran la seguridad alimentaria en hogares y d) que en los resultados se hiciera mención a hallazgos específicos sobre los hijos. La primera selección de textos fue de 58 trabajos, de los cuales se descartaron 10 porque la fecha de publicación excedía 5 años de antigüedad. Mediante una primera lectura rápida de los trabajos se descartaron otros 24 textos por dos razones: la primera, que ya se tenía una concentración de trabajos que podrían ubicarse en el enfoque biomédico, y la segunda causa de exclusión fue que, pese al gran número de trabajos antropológicos y socioculturales sobre la alimentación, en la gran mayoría no se hacía referencia a la seguridad alimentaria como marco analítico más amplio, siendo este el eje de reflexión de este trabajo.

Los 24 trabajos académicos que resultaron seleccionados para su análisis fueron sistematizados en matrices comparativas, en las cuales cada columna hacía referencia a un elemento de interés y las filas a los artículos revisados. Los aspectos contrastados fueron: sujetos o grupos de estudio, causas de la inseguridad alimentaria, consecuencias de la inseguridad alimentaria, definición utilizada, fuente de información y metodología, hallazgos principales y vacíos de investigación señalados por el autor o autores del trabajo. 


\section{Desarrollo y discusión}

La presente revisión bibliográfica se realizó con base en 24 trabajos académicos. Los artículos revisados se organizaron en torno a cuatro enfoques teóricos principales: el enfoque neoclásico, el enfoque biomédico, el enfoque sociológico y el enfoque de modos de vida sostenibles.

El enfoque neoclásico de la seguridad alimentaria parte de los conceptos de producción, oferta, demanda, precios de los alimentos, déficit de la oferta y déficit de la producción de alimentos. Bajo este enfoque la seguridad alimentaria se explica por la oferta de alimentos y la demanda efectiva en un tiempo determinado. A su vez, la disponibilidad de alimentos depende del nivel en los suministros alimentarios (incluidos los compuestos de la producción de subsistencia y los suministros del mercado procedentes de la producción interna, las existencias y las importaciones de alimentos); mientras que el acceso a los alimentos está determinado por la capacidad para expresar las necesidades alimentarias como demanda efectiva ( $\sin$ considerar la producción de subsistencia). En tanto que la estabilidad se refiere a las menores variaciones en la producción, los suministros y/o la demanda de alimentos a lo largo del tiempo (FAO, 2011).

Las investigaciones realizadas con base en este enfoque definen a la seguridad alimentaria como una situación en que la oferta y la demanda de alimentos son suficientes para atender las necesidades alimentarias de manera continua y estable; en contraste, la inseguridad alimentaria se define como las variaciones ocasionales, recurrentes $o$ permanentes en el volumen de la oferta o demanda de alimentos, siendo estos insuficientes para atender las necesidades de la población (déficit de la producción de alimentos o de la demanda debido a la carencia de recursos económicos) (FAO, 1999).

Algunos de los supuestos teóricos que se pueden inferir de este enfoque son:

- Los precios del mercado de los alimentos determinan el volumen de la producción, la oferta y la demanda en un país.

- Los cambios en los parámetros se producen como consecuencia del desarrollo económico o de medidas normativas específicas.
- Las personas consumen los alimentos en función de la elección racional que hace de los productos que se ofertan en el mercado y de su nivel de ingresos.

- Existe inseguridad alimentaria cuando la demanda efectiva supera la producción interna de alimentos y no existen importaciones.

- Existe inseguridad alimentaria cuando aun habiendo oferta, los consumidores tienen ingresos insuficientes para adquirir los alimentos y satisfacer sus necesidades.

En este enfoque se pueden ubicar trabajos como los de Sosa (2017) y Candela (2016). Sosa estima que, en México, debido al incremento poblacional de los últimos 35 años, la producción agrícola ha disminuido, debido a la disminución del total de hectáreas per cápita cultivadas (Sosa, 2017). Con su estudio concluyó que entre 1980 y el año 2015 la población aumentó 79 por ciento y la tierra agrícola disponible per cápita descendió en un 31 por ciento (Sosa, 2017). A decir de este autor, el país deberá introducir mejoras tecnológicas para potencializar el rendimiento de la superficie cultivable y poder asegurar el abasto de alimentos a los mexicanos hacia el año 2050, de lo contrario, ocurrirá una crisis alimentaria.

De acuerdo con un estudio del Banco Mundial (2015), en Brasil, el sector agrícola contribuye con $23 \%$ del Producto Interno Bruto, 33\% de los empleos y $43 \%$ de las exportaciones del país. Sin embargo, más del $1 \%$ del PIB se pierde debido a eventos extremos que no son atendidos oportunamente, poniendo en riesgo la producción de alimentos a nivel mundial para los próximos años. Mientras que Candela (2016) al analizar la situación venezolana concluyó que la disponibilidad de alimentos desde el año 2013 dependió de la importación de estos, por lo cual, derivado de las restricciones y la crisis económica enfrentada en 2015, el abastecimiento de alimentos es deficiente, los ingresos se contrajeron y hubo cambios en los patrones de consumo alimentario (Candela, 2016). Por su parte, Rivero y Aliaga (2014) encontraron que en Bolivia no existía disponibilidad de alimentos, y no la lograrían hacia el año 2030 como se esperaría. Sus afirmaciones las sustentaron en el hecho de que en ese país se han priorizando las exportaciones y una cultura de bajo consumo de frutas y verduras; por lo que la inseguridad alimentaria en el país difícilmente se 
solucionaría con un incremento en la oferta de alimentos nutritivos.

Todos estos trabajos se han realizado con base en estadísticas de las hojas de balance de alimentos y sus sistemas de cuentas nacionales; permitiendo con ello identificar tendencias generales a nivel país sobre los niveles de seguridad alimentaria.

Por otra parte, el enfoque biomédico construido a lo largo de los siglos XVI y XVII en Europa) es propio de la biomedicina (Fernández, 2004; eminentemente biológico, técnico y positivista en el abordaje del fenómeno salud-enfermedad-atención. Así, la persona al ser considerada en clave anatomopatológica, se convierte en un objeto medible; mientras que las causas de las enfermedades al buscarse en el aspecto biológico son consideradas como problemas susceptibles de intervención clínica (Fernández, 2004). La atención a la salud de las personas en este modelo, se sustenta en tres principios: a) la búsqueda de alteraciones anatómicas subyacentes a una enfermedad, b) la indagación de las alteraciones fisiológicas y c) las investigaciones etiológicas (Fernández, 2004). Por lo que, la evidencia sobre algún problema de inseguridad alimentaria se basa en la medición cuantitativa y biológica de problemas antropométricos y de salud que se pueden curar.

Fuertemente influenciados por el enfoque biomédico, gran parte de los estudios relacionados con la inseguridad alimentaria se enfocan en los resultados de ésta. En el caso de la población infantil, desde 1990, la estrategia mundial para combatir la malnutrición de los niños que lanzó el Fondo de las Naciones Unidas para la Infancia (UNICEF por sus siglas en inglés) planteó que la malnutrición es el resultado del "consumo inadecuado de nutrientes o por factores de enfermedad que afectan la digestión, absorción, transporte y utilización de nutrientes" (UNICEF, 1990); enfocándose en los aspectos medibles de las alteraciones biológicas evitables.

De hecho, durante muchos años, la ingesta calórica fue considerada el estándar para medir el acceso a los alimentos a nivel doméstico, y a nivel individual las medidas antropométricas del estado nutricional; sin embargo, ambos tipos de acceso están determinados por diversos factores. La ingesta calórica per cápita refleja el consumo actual de alimentos, pero no considera otros aspectos como son: la calidad, diversidad dietética, suficiencia de micronutrientes, vulnerabilidad, riesgos, fluctuaciones en el tiempo, etcétera. No obstante, las medidas antropométricas se usan con mayor frecuencia porque su medición es más barata (Maxwell, Coatles y Vaitla, 2013).

Dentro de los estudios recientes sobre la seguridad alimentaria de hogares con población menor de 18 años, la unidad de análisis ha sido el hogar, y la mayoría se enfocan en los menores de cinco años (Papas, Trabulsi, Dahl y Dominick, 2015; Urquía, 2014; Vega, et al, 2014; Cuevas, et al, 2014; Mundo, et al, 2014; Mundo, Shamah y Rivera, 2013). Este mayor énfasis en los menores de cinco años se debe a la consideración de que ellos son el grupo de población más vulnerable en situaciones de inseguridad alimentaria y donde pueden identificarse consecuencias de más largo plazo.

Estos autores sitúan las causas de la inseguridad alimentaria en la ingesta inadecuada de micronutrientes, los escasos recursos económicos de los hogares, y el limitado acceso a dietas adecuadas en nutrientes y energía; factores que ocasionan comidas de baja calidad, desnutrición, depresión y cambios en sus hábitos alimenticios. En el caso de México, los estudios realizados, con enfoques cuantitativos, identificaron que los niños de hogares mexicanos con inseguridad alimentaria severa eran aquellos que se encontraban en condiciones de pobreza, con jefatura indígena, familias grandes, jefes de hogar con bajo nivel educativo o femenina; y que debido a la baja diversidad alimentaria (menor consumo de frutas, verduras, fibra y energía), presentaban desnutrición crónica y baja talla para la edad.

En el contexto estadounidense, Papas, Trabulsi, Dahl y Dominick (2015), así como Kaur y colaboradores (2015), encontraron que la ingesta inadecuada o reducida de alimentos en niños ocasiona un déficit del desarrollo cognitivo, conductas disfuncionales, tensiones emocionales y alteraciones en los patrones de alimentación con tendencia a la obesidad. Además, de que la inseguridad alimentaria era causada por los bajos ingresos económicos, el tamaño y la presencia de varios niños en el hogar.

Otros trabajos que se enfocaron en el estudio de los hogares en general, sin distinguir la edad de sus 
integrantes, también encontraron que algunas de las causas de la inseguridad alimentaria son: la presencia de población dependiente, como son niños y adultos mayores; la ausencia de redes de apoyo social (Valencia y Ortíz, 2014); la disponibilidad o no de activos en el hogar (tierras para cultivo) (Zárate, et al., 2015); no pertenecer a algún sistema de seguridad social (Castro y Camberos, 2017); así como el habitar en localidades aisladas y carentes de accesos carreteros (Haro, et al., 2016). Además, identificaron que, en los hogares, el recibir ayuda de programas sociales poseer tierras para cultivo de alimentos o el que alguno de sus miembros tenga empleo remunerado no significa que los hogares tendrán suficientes ingresos para adquirir alimentos.

Entre las líneas de investigación sobre las cuales los investigadores consideran se debe profundizar el trabajo en el tema de seguridad alimentaria se encuentran:

- Los estudios comparativos y longitudinales a partir de diferentes medidas de seguridad alimentaria y de medidas antropométricas.

- La cantidad real y calidad de los alimentos que consumen los integrantes de un hogar

- El peso de la variable condición indígena del hogar.

- Los efectos de los programas sociales que reciben los hogares.

- Las estrategias de subsistencia de los hogares en caso de sufrir inseguridad alimentaria.

- La prevalencia de enfermedades respiratorias, crónicas no transmisibles y diarreicas en los hogares con inseguridad alimentaria.

- Las prácticas de inocuidad alimentaria de los hogares.

- Las preferencias culturales por la alimentación y su efecto sobre la inseguridad alimentaria, y

- Las fluctuaciones locales de los precios de los alimentos y su efecto sobre el consumo de los hogares.

Un tema poco estudiado en las investigaciones del enfoque biomédico fue el de la inseguridad alimentaria y sus consecuencias para las jefas del hogar. McDonald y colaboradores (2015), estudiaron la inseguridad alimentaria de madres e hijos en Camboya como causal de desnutrición. Entre sus hallazgos destaca la importante prevalencia de delgadez y malnutrición materna, debido a la asignación diferenciada e injustificada de alimentos dentro del hogar. A decir de los autores, las encuestas de percepción de inseguridad alimentaria no permiten identificar si dentro de los hogares se priorizaban las necesidades nutricionales de los niños o de otros miembros del hogar en detrimento de las madres; sin embargo, dan cuenta de un problema que en otros estudios se menciona someramente o incluso se invisibiliza.

Desde el punto de vista metodológico, es importante mencionar que en la mayoría de los estudios revisados se encuentra ausente un enfoque teórico, tanto general como específico, en torno al cual se articulen las reflexiones sobre la seguridad alimentaria. Con un fuerte perfil positivista y biomédico se enfocan en dar cuenta de prevalencias de enfermedades de malnutrición o de percepciones de inseguridad alimentaria, sin profundizar en el carácter multidimensional de este problema social. Este vacío teórico se evidencia con las escazas referencias conceptuales sobre lo que entienden los autores por seguridad alimentaria. Además, la mayoría de los estudios se han realizado a partir de grandes encuestas nacionales de salud o seguridad alimentaria y con base en las escalas mundiales sobre percepciones de seguridad alimentaria de los hogares, a través de historiales de frecuencias de alimentos consumidos, medidas antropométricas y patrones de gasto. Sin embargo, hay escasas referencias sobre grupos poblacionales específicos (personas con discapacidad, migrantes, adultos mayores e indígenas).

El tercer enfoque teórico identificado es el sociológico o de la sociocultura alimentaria. Con raíces antropológicas estructural-funcionalistas, este tipo de estudios parten de la consideración de que la nutrición tiene dos funciones, una biológica y una social; por lo que se enfocan en analizar los comportamientos alimentarios y los modos de comer como indicadores de estatus social (Díaz y Gómez, 2005); así como el carácter expresivo de las representaciones entorno a la alimentación. Uno de los teóricos más influyentes en este enfoque es Pierre Bourdieu, para quien los cambios en las preferencias alimentarias se explican por los habitus, es decir, las prácticas y representaciones de un grupo social determinado en torno a la alimentación; 
Tabla 1. Investigaciones sobre seguridad alimentaria desde el enfoque biomédico

\begin{tabular}{|c|c|c|c|c|c|c|c|}
\hline Trabajo & Pais & $\begin{array}{l}\text { Unidad } \\
\text { de } \\
\text { Análisis }\end{array}$ & $\begin{array}{l}\text { Sujetol } \\
\text { grupo de } \\
\text { estudio }\end{array}$ & $\begin{array}{c}\text { Causas de la Inseguridad } \\
\text { Alimentaria (IA) }\end{array}$ & $\begin{array}{l}\text { Consecuencias de la } \\
\text { Inseguridad Alimentaria } \\
\text { (IA) }\end{array}$ & Metodología & Prevalencia de IA \\
\hline $\begin{array}{l}\text { Papas, M., Trabulsi, J., Dahl, A, y } \\
\text { Dominiok, G., (2015). }\end{array}$ & $\begin{array}{l}\text { Estados } \\
\text { Unidos, } \\
\text { Pensilvania }\end{array}$ & Hogares & $\begin{array}{l}\text { Madrese } \\
\text { hijos de } \\
\text { entre } 2 \text { y } 8 \\
\text { años de } \\
\text { edad }\end{array}$ & $\begin{array}{l}\text { Género, nivel de ingres os } \\
\text { bajos, edad, raza, etnicidad }\end{array}$ & $\begin{array}{l}\text { Obesidad, enfermedades } \\
\text { cardioves aulares, diabetes, } \\
\text { enfermedades mentales y } \\
\text { físicas. }\end{array}$ & $\begin{array}{l}\text { Entrevis tas y encuestas a } \\
\text { pacientes de una dínica para } \\
\text { his panos en Pens ilvania. } \\
\text { USA. Medidas } \\
\text { antropométricas. }\end{array}$ & $\begin{array}{c}74 \% \text { de hogares } \\
\text { con inseguridad } \\
\text { alimentaris }\end{array}$ \\
\hline $\begin{array}{l}\text { URQUíA, N., (2014). "La seguridad } \\
\text { al imentaria en México". }\end{array}$ & México & Hogares & $\begin{array}{l}\text { Niños } \\
<5 \text { años }\end{array}$ & $\begin{array}{l}\text { Diferenciales en el acoeso a } \\
\text { alimentos en los grupos de } \\
\text { población. }\end{array}$ & $\begin{array}{l}\text { No adecuado des arrollo físico } \\
\text { e intelectual. Des nutrición } \\
\text { a ónica infantil. } \\
\text { > prevalencia de sobrepes o y } \\
\text { obesidad. }\end{array}$ & $\begin{array}{l}\text { ENSANUT. Balanza } \\
\text { comercial, Unidades } \\
\text { Económicas Rurales. } \\
\text { Tratamiento es tadistico de la } \\
\text { información recopilada. }\end{array}$ & $\begin{array}{l}1 / 4 \text { de los hogares } \\
\text { mexicanos }\end{array}$ \\
\hline $\begin{array}{l}\text { VEGA M., Shamah, T., Peinador, } \\
\text { R., Méndez, I., y Melgar, H. (2014). }\end{array}$ & México & Hogares & $\begin{array}{l}\text { Niños <5 } \\
\text { años } \\
\text { Niños }<5\end{array}$ & $\begin{array}{l}\text { Nivel educativo de la madre, } \\
\text { condición indigena del hogar }\end{array}$ & $\begin{array}{l}\text { Des nutrición. Dietas de } \\
\text { menor calidad }\end{array}$ & $\begin{array}{l}\text { ENIGH 2008. Tr atamiento } \\
\text { estadístico de la información. } \\
\text { ENSANUT 2012, Escala }\end{array}$ & $\begin{array}{l}48 \% \text { de hogares } \\
\text { con IA }\end{array}$ \\
\hline $\begin{array}{l}\text { CUEVAS, L., Rivera, J., Shamah, } \\
\text { T., Mundo, V., y Ménder, L. (2014). }\end{array}$ & México & Hogares & $\begin{array}{l}\text { años } \\
\text { (prees colare } \\
\text { s) }\end{array}$ & & Desnutriaón. & $\begin{array}{l}\text { ELCSA Índices } \\
\text { antropométricos. Tratamiento } \\
\text { estadistico de la información. }\end{array}$ & $\begin{array}{c}31 \% \text { de hogares } \\
\text { con IA }\end{array}$ \\
\hline $\begin{array}{c}\text { MUNDO, V., Cruz, V., Jiménez, A, } \\
\text { y Shamah, T., }(2014) .\end{array}$ & México & Hogares & $\begin{array}{l}\text { Niños }<5 \\
\text { años }\end{array}$ & $\begin{array}{l}\text { Restricciones económicas. } \\
\text { Aumento en precio de los } \\
\text { alimentos. }\end{array}$ & $\begin{array}{c}\text { Cambios en los hábitos } \\
\text { alimentioios. }\end{array}$ & $\begin{array}{l}\text { ENSANUT 2012, Escala } \\
\text { ELCSA, Tratamiento } \\
\text { estadistico de la información. }\end{array}$ & $\begin{array}{c}33.7 \% \text { de hogares } \\
\text { con IA moder ada y } \\
\text { severa }\end{array}$ \\
\hline $\begin{array}{c}\text { MUNDO, V., Shamah, T.. Rivera, } \\
\text { JA, (2013). }\end{array}$ & México & Hogares & $\begin{array}{l}\text { Niños }<5 \\
\text { años }\end{array}$ & & $\begin{array}{l}\text { Depresión. Problemas de } \\
\text { comportamiento. < consumo } \\
\text { de micronutientes. Baja } \\
\text { Talla, anemia, sobrepeso, } \\
\text { obes idad, enfermedades } \\
\text { cónico degener ativas. }\end{array}$ & $\begin{array}{l}\text { ENSANUT 2012, Escala } \\
\text { ELCSA Índices } \\
\text { antropométricos. Tratamiento } \\
\text { estadistico de la información. }\end{array}$ & $\begin{array}{c}28.2 \% \text { de hogares } \\
\text { con IA moderada o } \\
\text { severa }\end{array}$ \\
\hline $\begin{array}{l}\text { KAUUR, J., Lamb, M., y Ogden, C. } \\
\qquad(2015) .\end{array}$ & USA & Hogares & $\begin{array}{c}\text { Niños de } 2 \text { a } \\
11 \text { años }\end{array}$ & $\begin{array}{l}\text { Escas os recurs os } \\
\text { económicos, acceso limitado } \\
\text { a alimentos, y condición ética } \\
\text { de los hogares. }\end{array}$ & $\begin{array}{l}\text { Alteraciones en los patrones } \\
\text { de alimentación, menor } \\
\text { calidad de las dietas. }\end{array}$ & $\begin{array}{l}\text { The National Health and } \\
\text { Nutrition Examination Survey }\end{array}$ & $\begin{array}{c}9.8 \% \text { de niños con } \\
\text { IA }\end{array}$ \\
\hline VALENCIA, R., y Ortiz, L (2014). & México & Hogares & Hogares & $\begin{array}{c}\text { Riqueza material, } \\
\text { características } \\
\text { sociodemográficas de jefe de } \\
\text { hogar, ingres os, composición } \\
\text { del hogar, s in redes, no } \\
\text { protección social. Precio de } \\
\text { los alimentos. }\end{array}$ & $\begin{array}{l}\text { Menor dis ponibilidad de } \\
\text { futas y verduras fres cas, } \\
\text { carnes magras, láctecs y } \\
\text { bebidas azucaradas en } \\
\text { hogares con IA }\end{array}$ & $\begin{array}{l}\text { Encuesta Nacional de } \\
\text { Ingreso y Gas to de los } \\
\text { Hogares (ENIGH) } 2010\end{array}$ & $\begin{array}{l}28 \% \text { de hogares } \\
\text { con niños con IA } \\
\text { moderada y severa. }\end{array}$ \\
\hline $\begin{array}{l}\text { ZÁRATE, GS., Méndez, JA., } \\
\text { Ramirez, J., y Olvera, JI., (2016). }\end{array}$ & $\begin{array}{l}\text { México. } \\
\text { Municipio } \\
\text { de Puebla. }\end{array}$ & Hogeres & Hogares & $\begin{array}{c}\text { Activos (tierras dis ponibles } \\
\text { en el hogar). Choques } \\
\text { externos. } \\
\text { Características }\end{array}$ & $\begin{array}{c}\text { Suficiencia alimentar ia } \\
\text { deficiente. }\end{array}$ & $\begin{array}{l}\text { ENCUESTA en hogares, } \\
\text { utilización de es cala EMSA }\end{array}$ & $\begin{array}{c}40 \% \text { de hogares } \\
\text { con IA moder ada y } \\
\text { severa. }\end{array}$ \\
\hline Castro y Cambrero, (2017). & $\begin{array}{l}\text { México, } \\
\text { Sonora }\end{array}$ & Hogares & $\begin{array}{l}\text { Miembros de } \\
\text { los hogares }\end{array}$ & $\begin{array}{l}\text { sociodemográficas, pobreza } \\
\text { por ingresos, sin empleo } \\
\text { seguro, sin seguridad social. }\end{array}$ & & Encuesta Intercensal 2015. & $\begin{array}{c}27.8 \% \text { pobladión } \\
\text { con IA }\end{array}$ \\
\hline $\begin{array}{c}\text { HARO, R., Mar celeño, S., } \\
\text { Bojórquez, J., y Nájera, O.. (2018). }\end{array}$ & $\begin{array}{l}\text { México, } \\
\text { Nayarit }\end{array}$ & Hogares & Hogares & $\begin{array}{l}\text { Condioiones de la localidad } \\
\text { de res idencia, mercado } \\
\text { laboral precario, bajos } \\
\text { ingresos. }\end{array}$ & & Encuesta probabilística & $\begin{array}{c}25.55 \text { de hogares } \\
\text { con IA moder ada y } \\
\text { severa. }\end{array}$ \\
\hline $\begin{array}{l}\text { MoDONALD, CM., Mclean, J., } \\
\text { Kroeun, H., Talukder, A., Lynd, iD, } \\
\text { y Green, TJ., }(2015) \text {. }\end{array}$ & Camboya & Hogares & $\begin{array}{l}\text { Madres e } \\
\text { hijos }\end{array}$ & & $\begin{array}{l}\text { Malnutrioón y anemia de } \\
\text { hijos y madres. }\end{array}$ & $\begin{array}{c}\text { Encuesta y mediciones } \\
\text { antropomét icas }\end{array}$ & $\begin{array}{l}48.8 \% \text { de hogares } \\
\text { con IA moder ada y } \\
\text { severa }\end{array}$ \\
\hline
\end{tabular}

Fuente: elaboración propia. 
constituyéndose ésta en un criterio de diferenciación de clases sociales, prestigio, estatus, de distinción u ostentación (Díaz y Gómez, 2005).

Desde este enfoque es posible encontrar la idea de que la alimentación es generadora de opiniones creencias y significados que inciden en la producción, distribución, selección, preparación y consumo de alimentos. Esta consideración ha dado lugar a estudios interesados en dar cuenta de la construcción social de la alimentación y los factores asociados (entre los que se encuentran: la cultura alimentaria, las costumbres familiares, la publicidad, las relaciones de poder dentro de los hogares, los aspectos subjetivos que se vehiculizan en los alimentos y a través de la experiencia con estos); sin embargo, el común denominador de los estudios es la escasa o nula consideración de estas preferencias como factores asociados a la seguridad alimentaria de los hogares y las personas.

Arboleda y Villa (2016) afirman que la alimentación es un hecho y acción que determina además de lo biológico, a las diferentes identidades, las condiciones de bienestar y la auto-representación de los sujetos. Además, afirman que las preferencias por determinados alimentos están permeadas por significados sobre los alimentos, las preparaciones y las experiencias. Arboleda (2014) y Arboleda y Villa (2016), consideran que las situaciones de escasez de alimentos contribuyen a la configuración de estrategias familiares para acceder a la alimentación del grupo familiar. Las estrategias que las familias adoptan pueden ser: a) elegir la compra de alimentos más baratos y saciadores, b) consumir aquello que se requiere (por obligación) en situaciones de enfermedad, c) consumir lo que sea práctico, sin importar contenido nutricional, d) disminuir el número de comidas al día, e) rebuscar en los desechos, la mendicidad o buscar redes de apoyo, f) servir porciones más pequeñas en las comidas principales, g) ingerir una bebida para calmar el hambre y h) cambiar los horarios habituales de las comidas y de sus actividades.

Otros estudios entorno a la alimentación señalan que, ésta depende las prácticas y representaciones que socialmente han construido en torno a la salud y la salubridad de sus viviendas. Assunta y colaboradores (2016) encontraron que las prácticas alimentarias en familias chilenas estaban fuertemente afectadas por las prácticas higiénico-sanitarias del hogar. En el mismo sentido, un estudio de la UNICEF en Guatemala, Honduras y El Salvador, identificó la deficiente salubridad e higiene de los hogares, con importantes consecuencias para la salud de los niños; pero no como causal de inseguridad alimentaria, sino como variable característica del contexto de los hogares en esta condición. El mismo estudio da cuenta de cómo los hogares internalizan sus prácticas alimentarias con base en creencias culturales; por ejemplo, el que aseguren que las comidas frías pueden causar cambios de temperatura física en el cuerpo, dolor de estómago y diarrea; y que la desnutrición ocurre por actos de brujería, mal de ojo, tristeza o parásitos (UNICEF-SESAN, 2014).

Finalmente, un enfoque emergente en México y América Latina es el Enfoque de modos de vida sostenibles. Éste tiene su origen en la Comisión de Medio Ambiente y Desarrollo de Brundtland, y fue extendido hacia otros países después de la Conferencia de las Naciones Unidas sobre Medio Ambiente y Desarrollo de 1992 y creado con el objetivo de evaluar las estrategias de las personas en situaciones de pobreza (Valdés y Pérez, 2011). Por modos de vida sostenibles se entiende el conjunto de capacidades, bienes, capitales y actividades que realizan las personas para vivir; y son sostenibles cuando permiten hacer frente e incluso recuperarse de tendencias adversas o shocks repentinos, así como cuando permiten el mantenimiento y mejora de las propias capacidades y activos presentes y futuros sin socavar la base de recursos naturales (FAO, 2009, citado en Valdés y Pérez, 2011). Entre los principales modelos del enfoque de modos de vida sostenibles se encuentran: el del Programa de las Naciones Unidas para el Desarrollo (PNUD), el modelo del Departamento para el Desarrollo Internacional (DFID) del Reino Unido, el del Fondo Internacional para el Desarrollo Agrícola (FIDA), el programa de Asistencia Cooperativa (CARE) y el de la Organización de Alternativas de Desarrollo (DA) (Valdés y Pérez, 2011).

El planteamiento principal este enfoque es que las familias se encuentran insertas en contextos de vulnerabilidad, y que poseen activos cuyo valor depende del entorno social, institucional y organizativo. Con base en éste, Pat y colaboradores (2011) encontraron que las diferencias en las estrategias familiares para hacer frente a los choques 
externos que afectan el consumo y producción agrícola de alimentos se explican por los activos y el capital social que poseen (la tenencia de tierras mecanizadas, la afiliación a organizaciones productivas y los subsidios productivos recibidos). Son los capitales los que también establecen las diferencias en las capacidades de las comunidades para aumentar o reducir sus condiciones de vida, siendo el capital natural el más importante. Además, los estudios revisados señalan que los hogares que destinan un alto porcentaje del ingreso del hogar a la compra de alimentos se convierten en unidades domésticas con inseguridad alimentaria, ya que esto no les permite ahorrar, mejorar las condiciones de su vivienda y mucho menos invertir en la mecanización de sus tierras, además de consumir dietas poco variadas (Pasquier, 2017; Zárate, et al, 2016, Román, et al., 2010, Serrano, et al., 2016).

En el estado de Chiapas, Pasquier (2017) identificó escenarios de inseguridad alimentaria crónica causados por: la escasez de maíz en algunas épocas del año, el aumento continuo de los precios de productos de primera necesidad, la interrupción en la transmisión generacional de conocimientos sobre el uso de los recursos naturales, el desplazamiento forzado, la inexistencia de vínculos sociales, las enfermedades de los miembros del hogar y las migraciones fallidas.

Si bien se trata de un enfoque poco utilizado, se considera que éste posee potencial analítico en las investigaciones sobre seguridad alimentaria, especialmente para comprender el contexto en el cual se encuentran los hogares, como éste les afecta o beneficia y como responden a sus efectos en la búsqueda cotidiana de satisfacer sus necesidades alimenticias. Además, se trata de un enfoque que permite visualizar hacia donde se pueden reorientar los apoyos gubernamentales para familias en condiciones de inseguridad alimentaria. Cabe señalar que este modelo enmascara procesos, comportamientos, decisiones, creencias, costumbres y tradiciones de los núcleos familiares, que definen e incluso determinan el cómo las familias acceden a los alimentos o cómo cubren sus necesidades.

\section{Conclusiones}

Combatir el hambre y garantizar la seguridad alimentaria y nutricional de la población forma parte de los Objetivos de Desarrollo Sostenible de la
Agenda 2030 a nivel mundial. Los estudiosos de la alimentación y nutrición están frente a uno de los principales problemas sociales a nivel internacional y por ende aún hay amplios campos de investigación en los cuales deberá profundizarse, no sólo en lo que a espacios geográficos estudiados se refiere, sino también a unidades de análisis poco estudiadas con mayor detalle, como son los niños y niñas mayores de 5 años, adultos mayores, migrantes, minorías étnicas y adolescentes, sólo por mencionar algunos.

Como se mostró a lo largo de esta revisión, la mayor cantidad de estudios sobre seguridad alimentaria se han realizado desde un enfoque biomédico y con el objetivo de mostrar aspectos específicos de la inseguridad alimentaria, como son: la ingesta calórica individual, las alteraciones anatómicas y de la salud, y la percepción de las familias sobre su acceso, disponibilidad y suficiencia de alimentos. Sin embargo, estos requieren de un enfoque teórico más amplio que vaya más allá de las estadísticas y mediciones antropométricas que utilizan. Los estudios sociológicos en torno a la alimentación, muy centrados en el trabajo cualitativo también requieren ampliar sus discusiones hacia la seguridad alimentaria de las comunidades o grupos sociales de estudio, como puede ser el efecto de las estrategias de los hogares en situaciones de escasez de alimentos sobre el estado nutricional de sus integrantes. Finalmente, los trabajos realizados a partir del enfoque de modos de vida sostenibles, aunque es emergente, también posee un potencial analítico que puede explotarse en futuras investigaciones, tomando en cuenta que debido a los factores que considera tiene mayor aplicabilidad en contextos geográficos pequeños.

Los enfoques teóricos, los aspectos, determinantes y consecuencias de situaciones y condiciones de inseguridad alimentaria que se identificaron en esta revisión bibliográfica dan cuenta de que se trata de un problema social multifacético y multidimensional; el cual no puede ser estudiado en su totalidad por una única teoría o una sola forma de aproximación empírica, ya que los marcos de investigación no son permanentes ni ahistóricos, sino que se configuran y reconfiguran al estar contextualizados espacio-temporalmente,

La complejidad de la seguridad alimentaria y nutricional de la población humana exige avanzar 
hacia la construcción de modelos de análisis complejos que permitan contar con nuevas formas de ver la realidad, de propiciar el diálogo inter y multidisciplinario y con ello superar reduccionismos teóricos y metodológicos. Continuar los estudios sobre seguridad alimentaria y nutricional sólo desde un enfoque teórico o una metodología específica conlleva el riesgo de cosificar el concepto, resultando inevitable la cuantificación y descripción, y menos el contribuir desde la ciencia a mejorar las condiciones de vida de las personas.

\section{Bibliografía}

Arboleda, L. (2014). Dinámicas y estrategias alimentarias instauradas en hogares de Medellín. Revista Facultad Nacional de Salud Pública, 32 (3), 282-289. Recuperado de http://www.scielo.org.co/pdf/rfnsp/v32n3/v32n3a03.p df

Arboleda, L. \& Villa, P. (2016). Preferencias alimentarias en los hogares de la ciudad de Medellín, Colombia. Saúde e Sociedade, 25(3), 750-759. Recuperado de http://www.scielo.br/pdf/sausoc/v25n3/1984-0470sausoc-25-03-00750.pdf

Assunta, B., Souza G., Paz, C. \& Pozzagnol, M. (2016). Condiciones socioeconómicas e higiénico-sanitarias como dimensiones de la seguridad alimentaria y nutricional. Revista chilena de nutrición, 43(1), 62-67. DOI: $10.4067 / \mathrm{S} 0717-75182016000100009$

Banco Mundial. (2015). Revisão rápida e integrada da gestão de riscos agropecuários no brasil Caminhos para uma visão integrada. Brasília: Banco Mundial. Recuperado http://documents.worldbank.org/curated/en/71756146 7986362017/pdf/AUS12876-PORTUGUESEREVISED-PUB-OUO-9-RiscosAgropecu\%C3\%A1rios-no-Brasil-World-BankGroup-paginas-compressed.pdf

Candela, Y. (2016). Seguridad alimentaria en Venezuela: una mirada desde el ciudadano vulnerable. Cuadernos del CENDES, 33 (91), 125-139. Recuperado de http://www.redalyc.org/articulo.oa?id=40347542008.

Castro, A., \& Camberos, M. (2017), "Pobreza alimentaria: inseguridad y vulnerabilidad en las regiones de Sonora en 2015", Iztapalapa. Revista de Ciencias Sociales y Humanidades, 38(83), 43-73. Recuperado de http://www.scielo.org.mx/pdf/izta/v38n83/20079176-izta-38-83-00043.pdf

Cuevas, L., Rivera, J., Shamah, T., Mundo, V., \& Méndez H. (2014). Inseguridad alimentaria y estado de nutrición en menores de cinco años de edad en México. Salud Pública de México, 56(1), 47-53. Recuperado de http://www.scielo.org.mx/scielo.php?script=sci_arttex t\&pid=S0036-36342014000700008\&lng=es\&tlng=es.

Díaz, C., Gómez, C., (2005). "Sociología y alimentación”, Revista Internacional de Sociología, [S.l.], v. 63, n. 40, pp. 21-46. Recuperado dehttp://revintsociologia.revistas.csic.es/index.php/rev intsociologia/article/view/188/239

FAO y OPS. (2017). Panorama de la Seguridad Alimentaria y Nutricional en América Latina y el Caribe. Santiago de Chile. Recuperado de: http://www.fao.org/3/a-i7914s.pdf

FAO. (1996). Cumbre Mundial sobre la Alimentación. Recuperado http://www.fao.org/docrep/X2051s/X2051s00.htm

FAO. (1999). Implicaciones de las políticas económicas en la seguridad alimentaria: Manual de capacitación. Recuperado de: http://www.fao.org/docrep/004/W3736S/W3736S00. HTM

FAO. (2011). Seguridad Alimentaria Nutricional, Conceptos Básicos 3ra Edición, febrero de 2011. Recuperado de http://www.fao.org/3/a-at772s.pdf

Fernández, G., (coordinador) (2004). Salud $e$ interculturalidad en América Latina. Perspectivas antropológicas. Quito-Ecuador: Abya-Yala ediciones. Recuperado https://www.ucursos.cl/medicina/2015/2/NU0102091 0010/1/material_docente/bajar?id_material=1246455

Haro, R., Marceleño, S., Bojórquez J. \& Nájera, O. (2016). $\mathrm{La}$ inseguridad alimentaria en el estado de Nayarit, México, y su asociación con factores socioeconómicos. Salud Pública de México, 58, 421-427.

Kaur, J., Lamb, M., \& Ogden, C. (2015). The association between food insecurity and obesity in children-The National Health and Nutrition Examination Survey. Journal of the Academy of Nutrition and Dietetics, 115(5), 751-758.

Maxwell, D., Coates, J., \& Vaitla, B., (2013). How Do Different Indicators of Household Food Security Compare? Empirical Evidence from Tigray. Feinstein International Center, Tufts University: Medford, USA. Recuperado de: http://fic.tufts.edu/assets/DifferentIndicators-of-HFS.pdf

Mcdonald, C., Mclean, J., Kroeun, H., Talukder, A., Lynd, L., \& Green, T. (2015). Household food insecurity and dietary diversity as correlates of maternal and child 
undernutrition in rural Cambodia. European Journal of Clinical Nutrition, 69(2), 242-246. DOI: 10.1038/ejcn.2014.161

Mundo V., Cruz V., Jiménez A., \& Shamah T. (2014). Diversidad de la dieta y consumo de nutrimentos en niños de 24 a 59 meses de edad y su asociación con inseguridad alimentaria. Salud Pública de México, 56, 39-46. Recuperado de http://www.scielo.org.mx/scielo.php?script=sci_arttex $\mathrm{t} \&$ pid=S0036-36342014000700007\&lng=es\&tlng=es.

Mundo, V., Shamah T., \& Rivera J. (2013). Epidemiología de la Inseguridad Alimentaria en México. Salud Pública de México, 55, 206-213. Recuperado de http://www.redalyc.org/articulo.oa?id=10628331018

Papas, M., Trabulsi, J., Dahl, A., y Dominick, G., (2015). Food Insecurity Increases the Odds of Obesity Among Young Hispanic Children. J, Immigrant Minority Health. DOI 10.1007/s10903-015-0275-0

Pasquier. A.G. (2017). La "seguridad alimentaria" desde un enfoque etnográfico. Estudio de caso en una comunidad de refugiados guatemaltecos en el estado de Chiapas. Cultura y representaciones sociales, 11(22), 131-157. Recuperado de http://www.scielo.org.mx/pdf/crs/v11n22/2007-8110crs-11-22-00131.pdf

Pat, L., Nahed J., Parra M., García L., Nazar A., \& Bello E. (2011). Influencia de las estrategias de ingresos y las políticas públicas sobre la seguridad alimentaria en comunidades rurales mayas del norte de Campeche, México. Tropical and Subtropical Agroecosystems, 14(1). Recuperado de http://www.redalyc.org/articulo.oa?id=93915703006.

Rivero, B. \& Aliaga J. (2014). Disponibilidad, consumo y utilización biológica de alimentos en Bolivia: análisis y perspectivas (1990-2030). Revista Latinoamericana de Desarrollo Económico, (22), 161-198. Recuperado de

http://www.scielo.org.bo/scielo.php?script=sci_arttext \&pid=S2074-47062014000200006\&lng=es\&tlng=es.

Román, S., Vázquez, P., Hernández, H., \& Balenteasesor, O. (2010). Seguridad alimentaria con enfoque en modos de vida sustentable en el municipio de Oxchuc, Chiapas. Tesis de Maestría. México: El Colegio de la Frontera Sur.

Serrano, M., Calderón F., Vargas S., López H., Antonio P., Martínez G., \& Salcido B. (2016). Características estructurales y productivas de hogares con diferente grado de seguridad alimentaria en Puebla. Agricultura, sociedad y desarrollo, 13(4), 547-563. Recuperado de http://www.scielo.org.mx/scielo.php?script=sci_arttex $\mathrm{t} \& \mathrm{pid}=\mathrm{S} 1870-54722016000400547 \& \operatorname{lng}=\mathrm{es} \& \mathrm{t} \operatorname{lng}=\mathrm{es}$

Sosa, A. (2017). La disponibilidad de alimentos en México: un análisis de la producción agrícola de 35 años y su proyección para 2050. Papeles de Población, 23(93), 207-230. Recuperado de http://www.redalyc.org/jatsRepo/112/11252977008/ht $\mathrm{ml} / \mathrm{index} . \mathrm{html}$

UNICEF \& SESAN, (2014). Factores socioculturales que inciden en la desnutrición crónica. Guatemala. Fondo de las Naciones Unidas para la Infancia -UNICEF- y Secretaría de Seguridad Alimentaria y Nutricional SESAN-. Recuperado de: http://www.sesan.gob.gt/wordpress/wpcontent/uploads/2017/03/Factores-socioculturalesque-inciden-enla-desnutricion-cronica.pdf

UNICEF, (1990). Strategy for improved nutrition of children and women in developing countries. New York: UNICEF. Recuperado dehttp://repository.forcedmigration.org/show_metadat a.jsp?pid=fmo:3066

Urquía, N. (2014). La seguridad alimentaria en México. Salud Pública de México, 56, 92-98. Recuperado de http://www.scielo.org.mx/scielo.php?script=sci_arttex t\&pid=S0036-36342014000700014\&lng=es\&tlng=es.

Valdés, O., \& Pérez, A. (2011). Sustainable livelihoods: an analysis of the methodology. Tropical and Subtropical Agroecosystems, 14(1), 91-99. Recuperado de http://www.redalyc.org/pdf/939/93915703007.pdf

Valencia, R. \& Ortíz, L. (2014). Disponibilidad de alimentos en los hogares mexicanos de acuerdo con el grado de inseguridad alimentaria. Salud Pública de México, 56, 154-164. Recuperado de http://www.scielo.org.mx/scielo.php?script=sci_arttex t\&pid=S0036-36342014000200003\&lng=es\&tlng=es.

Vega M., Shamah T., Peinador R., Méndez I., \& Melgar H. (2014). Inseguridad alimentaria y variedad de la alimentación en hogares mexicanos con niños menores de cinco años. Salud Pública de México, 56, 21-30. Recuperado de http://www.scielo.org.mx/scielo.php?script=sci_arttex t\&pid=S0036-36342014000700005\&lng=es\&tlng=es.

Zárate, G.S., Méndez, J.A., Ramírez, J., \& Olvera, JI., (2016). Análisis de la seguridad alimentaria en los hogares del municipio de Xochiapulco Puebla, México. Estudios Sociales, 47, Enero-Junio 2016. $67-$ $84 . \quad$ Recuperado de https://www.ciad.mx/estudiosociales/index.php/es/arti cle/view/309/196 